\title{
Energy-efficient Power Allocation based on worst-case performance optimization under channel uncertainties
}

\author{
Xin Song, Li Dong*, Xue Huang, Lei Qin and Xiuwei Han \\ Northeastern University, \\ Shenyang 110819, China \\ [e-mail: dongll0801@163.com] \\ *Corresponding author: Li Dong
}

Received July 23, 2019; revised November 25, 2019; revised August 28, 2020; accepted October 2, 2020; published November 30, 2020

\begin{abstract}
In the practical communication environment, the accurate channel state information (CSI) is difficult to obtain, which will cause the mismatch of resource and degrade the system performance. In this paper, to account for the channel uncertainties, a robust power allocation scheme for a downlink Non-orthogonal multiple access (NOMA) heterogeneous network (HetNet) is designed to maximize energy efficiency (EE), which can ensure the quality of service (QoS) of users. We conduct the robust optimization model based on worse-case method, in which the channel gains belong to certain ellipsoid sets. To solve the non-convex non-liner optimization, we transform the optimization problem via Dinkelbach method and sequential convex programming, and the power allocation of small cell users (SCUs) is achieved by Lagrange dual approach. Finally, we analysis the convergence performance of proposed scheme. The simulation results demonstrate that the proposed algorithm can improve total EE of SCUs, and has a fast convergence performance.
\end{abstract}

Keywords: Non-orthogonal multiple access technology, heterogeneous networks, energy efficiency, channel state information, robust power allocation, Dinkelbach method, sequential convex programming, Lagrange dual approach 


\section{Introduction}

$\mathbf{N}_{\text {owadays, with the explosive growth of traffic demand, such as the popularity of mobile }}$ terminal devices, communication networks are facing enormous challenges in improving system capacity [1]. The shortage of spectrum resource is also receiving more and more attention. The data analysis shows that the demand for traffic data volume is expected to be 500-1000 times higher than that in 2010 [2]. Facing to these problems, HetNets and NOMA technology are proposed to improve the spectral efficiency, respectively. In HetNets, it consists of macro base stations (MBSs) and multiple small base stations (SBSs), where SBSs include picocell BSs and femtocells BSs [3-5]. NOMA technology has a lower complexity at receiver and allows multiple users to share the same subcarrier on the power domain to improve the spectral efficiency, so it is considered as a key candidate for future access technology [6].

Resource allocation plays a very significant role in both HetNets and NOMA system. In [7], the joint power allocation and user scheduling is investigated for a NOMA system based on EE, in which the imperfect CSI is considered. The power allocation problem is studied in [2] for NOMA system, where the difference of convex programing is applied as a suboptimal algorithm. The optimal power allocation based on max-min rate proportional fairness and max-sum rate is found in [8]. In [6], power allocation and sub-channel assignment are considered in NOMA system, which are solved by geometric programing and matching algorithm, respectively. In [9], the power allocation is discussed for HetNets, in which the difference of two convex functions programing are performed to obtain the Karush-Kuhn-Tucker solution. A lower bound problem and a parameterized transformation are introduced to achieve the energy efficient power allocation in OFDMA HetNets [10]. In full-duplex OFDMA system, the power allocation, user pairing, subchannel allocation are achieved as a three-sided matching problem via a novel matching algorithm.Considering the application of NOMA technology in HetNets, the convex relaxation technology is adopted to allocate the power for a downlink NOMA HetNets [11]. In [12], the sequential convex programing is discussed to obtain the close-form solution of powers in NOMA HetNets aiming to improve the sum rate of small cells. Also, a distributed power allocation scheme is discussed, where NOMA technology is applied at MBS and SBSs, its goal is to maximize the total throughput of system [13]. In [14], Xu et al. investigate resource allocation scheme for an energy-cooperation enabled two-tier NOMA HetNet.

Based on the above research, most of the literatures are carried out under the assumption that the CSI is perfect. However, in the actual communication environment, due to the estimation bias of the channel, delay feedback, random fluctuation and quantization error, it will cause the uncertainty of parameters in the system [15-17]. Therefore, in communication systems, robust algorithm with partial CSI and imperfect CSI are gradually receiving more and more attention [18]. In [19], time-varying nature of wireless links and the dynamics of random are captured under the consideration of channel uncertainty. Resource allocation problem is investigated under bounded channel uncertainty for a downlink OFDMA HetNet, in which each cellular network only deploys one user, while the cross-tier interference between MBS and SCUs with the uncertainty links is ignored [20]. In [21], Xu et al discuss a robust power allcation scheme for cognitive radio networks. Robust power control is also investigated, where the dual decomposition theory is adopted to solve robust power control problem in a distributed manner [22]. In [23], the imperfect CSI condition is introduced for downlink 
NOMA HetNets, where power alloctions for small cell and SCUs are achieved. The rest of the paper is organized as follows. Section 2 shows the NOMA HetNet system model and section 3 transforms the optimization problem by bounding the uncertainty of channel in ellipsoid uncertainty sets. In section 4, the sub-optimal power allocation algorithm is designed to achieve the power allocation for SCUs. The numerical results are shown in Section 5. And Section 6 conclude this paper.

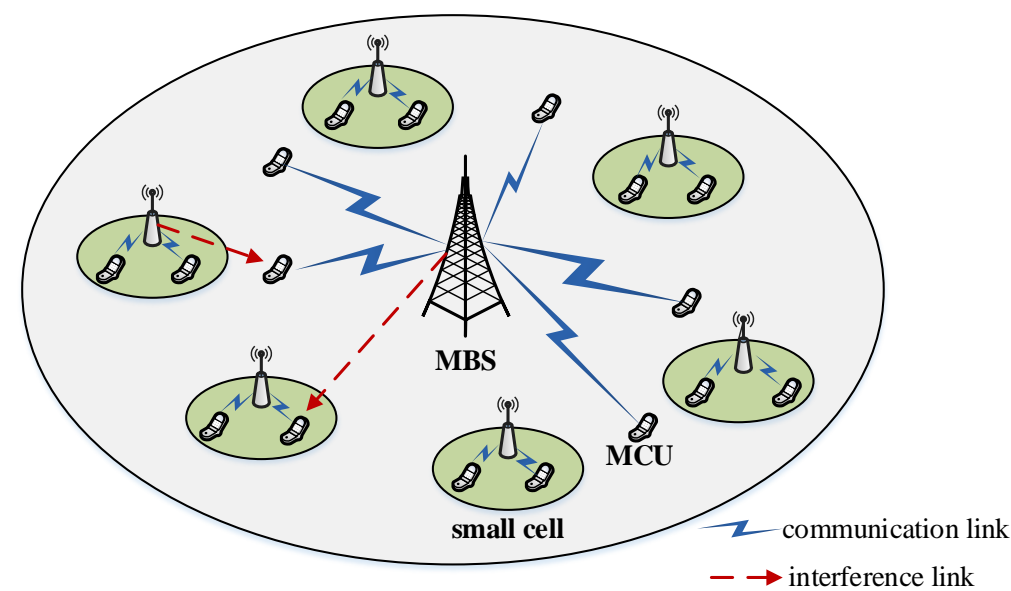

Fig. 1. NOMA HetNet System Model

\section{System Model}

\subsection{System Model}

Fig. 1 shows the system model for a downlink NOMA HetNet. It consists of a two-tier network, in which the upper layer represents the macro cell, and the lower layer represents the small cell. There are a MBS and $J$ macro cell users (MCUs) randomly distributed in the macro cell. The set of MCUs is defined as $\forall j \in \mathcal{J}=\{1,2, \ldots, J\}$. On the lower layer, there are $M$ small cells and $K$ SCUs which are randomly deployed within the coverage of the corresponding SBSs, here $\forall m \in \mathcal{M}=\{1,2, \ldots, M\}$ and $\forall k \in \mathcal{K}=\{1,2, \ldots, K\}$ denote the set of small cells and the set of small cell users, respectively. The NOMA technology is applied to this HetNet. Under this underlay spectrum sharing mode, SCUs are allowed to access the spectrum owned by MCUs. The system bandwidth is denoted by $W$. The $k$ th SCU in the $m$ th small cell is represented as $S C U_{m, k}$. The received signals of $S C U_{m, k}$ are expressed as

$$
y_{m, k}=h_{m, k} p_{m, k} s_{m, k}+h_{m, k} \sum_{k^{\prime}=1, k^{\prime} \neq k}^{K} p_{m, k^{\prime}} s_{m, k^{\prime}}+\sum_{m^{\prime}=1, m^{\prime} \neq k}^{M} g_{m, k}^{m^{\prime}} \sum_{k^{\prime}=1}^{K} p_{m^{\prime}, k^{\prime}} s_{m^{\prime}, k^{\prime}}+f_{m, k} \sum_{j=1}^{J} p_{j^{\prime}} s_{j}+\xi_{m, k}
$$

where the received signals of $S C U_{m, k}$ consist of the desired signal, the intra-tier interference from the other NOMA users, the co-tier interference from other small cells, the cross-tier interference from macro cell and the additive white Gaussian noise (AWGN). $h_{m, k}, h_{m, k}^{m}$ and $f_{m, k}$ are the channel coefficients of $S C U_{m, k}$ from the $m$ th SBS, $m$ th SBS and MBS, respectively. $p_{m, k}, p_{m, k^{\prime}}$ and $p_{j}$ represent transmission powers of $S C U_{m, k}, S C U_{m, k^{\prime}}$ and the $j$ th 
MCU, severally. $s_{m, k}, s_{m, k^{\prime}}$ and $s_{j}$ are the transmission symbols from corresponding BS to its serving users, respectively, $\xi_{m, k}$ is AWGN with zero mean and variance $\delta$.

In NOMA system, SIC technology is applied to SCUs. As illustrated in [14], it is assumed that the order of the channel to inter-cell interference plus noise ratios (CINRs) is in descending order. Based on this order, the users can decode the signal from the other NOMA users in the same small cell with smaller CINR value. The received signal-to-interference-plus-noise (SINR) of $S C U_{m, k}$ is written as

$$
\gamma_{m, k}=\frac{h_{m, k} p_{m, k}}{h_{m, k} \sum_{k^{\prime}=1, k^{\prime} \neq k}^{k-1} p_{m, k^{\prime}}+\sum_{m^{\prime}=1, \mathrm{~m} \neq m}^{M} g_{m, k}^{m^{\prime}} \sum_{k^{\prime}=1}^{K} p_{m^{\prime}, k^{\prime}}+f_{m, k} \sum_{j=1}^{J} p_{j}+\delta}
$$

According to Shannon formula, the sum rate of SCUs is expressed as

$$
R(\mathbf{P})=\sum_{m=1}^{M} \sum_{k=1}^{K} r_{m, k}=\sum_{m=1}^{M} \sum_{k=1}^{K} W \log _{2}\left(1+\gamma_{m, k}\right)
$$

where we denote $\mathbf{P} \in R^{M \times K}$ as the collection of optimization variable $p_{m, k}$

\subsection{Optimization Problem Formulation}

The purpose of this paper is to optimize the EE of all SCUs, which is defined as the trade of sum rate of SCUs and total power assumption. The optimization problem is written as

$$
\begin{aligned}
& \max E_{\text {total }}=\frac{R(\mathbf{P})}{P_{c}+\sum_{m=1}^{M} \sum_{k=1}^{K} p_{m, k}}=\frac{\sum_{m=1}^{M} \sum_{k=1}^{K} W \log _{2}\left(1+\gamma_{m, k}\right)}{P_{c}+\sum_{m=1}^{M} \sum_{k=1}^{K} p_{m, k}} \\
& \text { s.t. } \quad C 1: \sum_{m=1}^{M} \sum_{k=1}^{K} f_{m, j} p_{m, k} \leq I_{t h r} \\
& \quad C 2: r_{m, k} \geq R_{\min } \\
& \quad \text { C3: } \sum_{k=1}^{K} p_{m, k} \leq P_{\max } \\
& \text { C4: } p_{m, k} \geq 0
\end{aligned}
$$

where $E_{\text {total }}$ is the total EE of SCUs. $P_{c}$ is additional circuit power assumption. $I_{t h r}$ is the total interference threshold from SBS to MCUs. $R_{\min }$ denotes the minimum data rate of SCUs. $P_{\max }$ represents the maximum transmission power of SBS. $C 1$ and $C 2$ ensure the QoS of MCUs and SCUs, respectively. C3 guarantees the maximum power constraint of SBSs. C4 makes sure that the power of SCU is non-negative.

\section{Robust optimization model}

However, in practical communication systems, random interference and channel estimation error will bring different uncertainties, which will result in certain deviations in the system parameters. This paper mainly focus on the uncertainty of channel gain due to the channel estimated deviation to construct the channel model. Based on robust optimization theory, the ellipsoid uncertainty set is often used in previous papers [19], [21]-[22] to approximate an uncertainly region. 


\subsection{Uncertainty Sets}

Based on the robust optimization theory, the channel uncertainty from SBS to MCUs in C1 of (4) is defined as the following bounded ellipsoid uncertainty set $R_{f}$ :

$$
R_{f}=\left\{\Delta f_{m, j} \mid f_{m, j}=\bar{f}_{m, j}+\Delta f_{m, j}: \sum_{m=1}^{M}\left(\Delta f_{m, j}\right)^{2} \leq\left(\varepsilon_{j}\right)^{2}\right\}
$$

Considering the channel estimated error, the transformed SINR of $S C U_{m, k}$ is shown as

$$
\gamma_{m, k}=\frac{p_{m, k}}{\sum_{k^{\prime}=1}^{k-1} p_{k^{\prime}, m}+\sum_{m^{\prime}=1, m^{\prime} \neq m}^{M} G_{m, k}^{m^{\prime}} \cdot \sum_{k^{\prime}=1}^{K} p_{m^{\prime}, k^{\prime},}+F_{m, k} \sum_{j=1}^{J} p_{j}+\delta_{m, k}^{\prime}}
$$

where the normalized channel gains can be defined as, respectively

$$
G_{m, k}^{m^{\prime}}=\frac{g_{m, k}^{m^{\prime}}}{h_{m, k}}, \quad F_{m, k}=\frac{f_{m, k}}{h_{m, k}}, \quad \delta_{m, k}^{\prime}=\frac{\delta}{h_{m, k}}
$$

The channel uncertainty of $G_{m, k}^{m \prime}$ is defined as the following bounded ellipsoid uncertainty set $R_{G}$ :

$$
R_{G}=\left\{\Delta G_{m, j} \mid G_{m, k}^{m^{\prime}}=\bar{G}_{m, k}^{m^{\prime}}+\Delta G_{m, k}^{m^{\prime}}: \sum_{m^{\prime}=1, m^{\prime} \neq m}^{M}\left(\Delta G_{m, k}^{m^{\prime}}\right)^{2} \leq\left(\eta_{m, k}\right)^{2}\right\}
$$

And the channel uncertainty set $R_{F}$ of $F_{m, k}$ can be also expressed as

$$
R_{F}=\left\{\Delta F_{m, j} \mid F_{m, k}=\bar{F}_{m, k}+\Delta F_{m, k}:\left(\Delta F_{m, k}\right)^{2} \leq\left(\delta_{m, k}\right)^{2}\right\}
$$

\subsection{Transformation of Optimization Problem}

The above channel uncertainty parameters are subject to an infinite constraint on the uncertainty set. Cauchy-Schwarz inequality and worst-case approach in [24] will be introduced to transform optimization problem. According to (5), define the interference parameters of constraint $C 1$ in (4) as follows

$$
\begin{aligned}
I & =\sum_{m=1}^{M} \sum_{k=1}^{K} f_{m, j} p_{m, k}=\sum_{m=1}^{M} \sum_{k=1}^{K}\left(\bar{f}_{m, j}+\Delta f_{m, j}\right) p_{m, k} \\
& =\sum_{m=1}^{M} \sum_{k=1}^{K} p_{m, k} \bar{f}_{m, j}+\sum_{m=1}^{M} \sum_{k=1}^{K} p_{m, k} \Delta f_{m, j} \\
& \leq \sum_{m=1}^{M} \sum_{k=1}^{K} p_{m, k} \bar{f}_{m, j}+\sqrt{\sum_{m=1}^{M}\left(\sum_{k=1}^{K} p_{m, k}\right)^{2}} \cdot \sqrt{\sum_{m=1}^{M}\left(\Delta f_{m, j}\right)^{2}} \\
& \leq \sum_{m=1}^{M} \sum_{k=1}^{K} p_{m, k} \bar{f}_{m, j}+\sum_{m=1}^{M} \sum_{k=1}^{K} p_{m, k} \cdot \varepsilon_{j} \\
& =\sum_{m=1}^{M} \sum_{k=1}^{K} p_{m, k}\left(\bar{f}_{m, j}+\varepsilon_{j}\right)
\end{aligned}
$$

According to (10), the constraint $C 1$ in (4) will be represented as

$$
I \leq \sum_{m=1}^{M} \sum_{k=1}^{K} p_{m, k}\left(\bar{f}_{m, j}+\varepsilon_{j}\right) \leq I_{t h r}
$$


Based on the bounded ellipsoid uncertainty set (8), the interference parameters in objective function of (4) can be transformed as

$$
\begin{aligned}
\sum_{m^{\prime}=1, m^{\prime} \neq m}^{M} G_{m, k}^{m^{\prime}} \sum_{k^{\prime}=1}^{K} p_{m^{\prime}, k^{\prime}} & =\sum_{m^{\prime}=1, m^{\prime} \neq m}^{M}\left(\bar{G}_{m, k}^{m^{\prime}}+\Delta G_{m, k^{\prime}}^{m^{\prime}}\right) \cdot \sum_{k^{\prime}=1}^{K} p_{m^{\prime}, k^{\prime}} \\
& =\sum_{m^{\prime}=1, m^{\prime} \neq m}^{M} \bar{G}_{m, k}^{m^{\prime}} \cdot \sum_{k^{\prime}=1}^{K} p_{m^{\prime}, k^{\prime}}+\sum_{m^{\prime}=1, m^{\prime} \neq m}^{M} \Delta G_{m, k}^{m^{\prime}} \cdot \sum_{k^{\prime}=1}^{K} p_{m^{\prime}, k^{\prime}} \\
& \leq \sum_{m^{\prime}=1, m^{\prime} \neq m}^{M} \bar{G}_{m, k}^{m^{\prime}} \cdot \sum_{k^{\prime}=1}^{K} p_{m^{\prime}, k^{\prime}}+\sqrt{\sum_{m^{\prime}=1, m^{\prime} \neq m}^{M}\left(\Delta G_{m, k}^{m^{\prime}}\right)^{2}} \cdot \sqrt{\sum_{m^{\prime}=1, m^{\prime} \neq m}^{M}\left(\sum_{k^{\prime}=1}^{K} p_{m^{\prime}, k^{\prime}}\right)^{2}} \\
& \leq \sum_{m^{\prime}=1, m^{\prime} \neq m}^{M} \bar{G}_{m, k}^{m^{\prime}} \cdot \sum_{k^{\prime}=1}^{K} p_{m^{\prime}, k^{\prime}}+\eta_{m, k} \sqrt{\sum_{m^{\prime}=1, m^{\prime} \neq m}^{M}\left(\sum_{k^{\prime}=1}^{K} p_{m^{\prime}, k^{\prime}}\right)^{2}} \\
& =\sum_{m^{\prime}=1, m^{\prime} \neq m}^{M}\left(\bar{G}_{m, k}^{m^{\prime}}+\eta_{m, k}\right) \sum_{k^{\prime}=1}^{K} p_{m^{\prime}, k^{\prime}}
\end{aligned}
$$

Based on the bounded ellipsoid uncertainty set (9), the another interference parameter in optimization function of (4) can be shown as

$$
\begin{aligned}
F_{m, k} \sum_{j=1}^{J} p_{j} & =\left(\bar{F}_{m, k}+\Delta F_{m, k}\right) \sum_{j=1}^{J} p_{j}=\bar{F}_{m, k} \cdot \sum_{j=1}^{J} p_{j}+\Delta F_{m, k} \cdot \sum_{j=1}^{J} p_{j} \\
& \leq \bar{F}_{m, k} \cdot \sum_{j=1}^{J} p_{j}+\sqrt{\Delta F_{m, k}^{2}} \cdot \sqrt{\left(\sum_{j=1}^{J} p_{j}\right)^{2}} \\
& \leq \bar{F}_{m, k} \cdot \sum_{j=1}^{J} p_{j}+\delta_{m, k} \cdot \sqrt{\left(\sum_{j=1}^{J} p_{j}\right)^{2}} \\
& =\left(\bar{F}_{m, k}+\delta_{m, k}\right) \sum_{j=1}^{J} p_{j}
\end{aligned}
$$

Because the noise $\delta$ is very small relative to channel parameters $h_{m, k}$, the influence of channel estimated error $\Delta h_{m, k}$ on $\delta$ can be ignored. The normalized $\delta$ can be transformed as

$$
\delta_{m, k}^{\prime}=\frac{\delta}{m, k}=\frac{\delta}{\bar{h}_{m, k}+\Delta h_{m, k}} \approx \frac{\delta}{\bar{h}_{m, k}}
$$

Furthermore, the SINR for $S C U_{m, k}$ can be reformulated as

where

$$
\bar{\gamma}_{m, k}=\frac{p_{m, k}}{\sum_{k^{\prime}=1, k^{\prime} \neq k}^{k-1} p_{m, k^{\prime}}+A_{m, k}}
$$

$$
A_{m, k}=\sum_{m^{\prime}=1, \mathrm{~m} \neq m}^{M}\left(\bar{G}_{m, k}^{m^{\prime}}+\eta_{m, k}\right) \sum_{k^{\prime}=1}^{K} p_{m^{\prime}, k^{\prime},}+\left(\bar{F}_{m, k}+\delta_{m, k}\right) \sum_{j=1}^{J} p_{j}+\delta_{m, k}^{\prime}
$$

Above all, the novel optimization problem will be reformulated as follows: 


$$
\begin{gathered}
\max \bar{E}_{\text {total }}=\frac{\bar{R}(\mathbf{P})}{P_{c}+\sum_{m=1}^{M} \sum_{k=1}^{K} p_{m, k}}=\frac{\sum_{m=1}^{M} \sum_{k=1}^{K} W \log _{2}\left(1+\bar{\gamma}_{m, k}\right)}{P_{c}+\sum_{m=1}^{M} \sum_{k=1}^{K} p_{m, k}} \\
\text { s.t. } \quad C 1: \sum_{m=1}^{M} \sum_{k=1}^{K} p_{m, k}\left(\bar{f}_{m, j}+\varepsilon_{j}\right) \leq I_{\text {thr }} \\
C 2: \bar{r}_{m, k} \geq R_{\min } \\
C 3: \sum_{k=1}^{K} p_{m, k} \leq P_{\max } \\
C 4: p_{m, k} \geq 0
\end{gathered}
$$

The optimization problem (17) with respect to $p_{m, k}$ is no-convex and NP hard. Therefore, we could not obtain the optimal power in a polynomial time. Considering the complexity of calculating, a reasonable sub-optimal power allocation algorithm is essential, which will be introduced in next section.

\section{Robust Power Allocation Under Channel Uncertainty}

The optimization function in (17) as a nonlinear fractional form is transformed to a linear form via sequential convex programming and Dinkelbach method. Then, the Lagrangian dual approach is adopted to derive the close-form solution of powers for SCUs.

\subsection{Transformation of the Optimization Problem}

The sequential convex programming approach [25] will be introduced at first. The data rate for $S C U_{k, n}$ can be represented as via the lower bound of inequality

$$
\bar{r}_{m, k}=W \log _{2}\left(1+\bar{\gamma}_{m, k}\right) \geq \bar{r}_{m, k}^{*}=W\left(\alpha_{m, k} \log _{2} \bar{\gamma}_{m, k}^{*}+\beta_{m, k}\right)
$$

The bound will be tight when $\bar{\gamma}_{m, k}=\bar{\gamma}_{m, k}^{*}$, where $\alpha_{m, k}=\frac{\bar{\gamma}_{m, k}^{*}}{1+\bar{\gamma}_{m, k}^{*}}$ and $\beta_{m, k}=\log _{2}\left(1+\bar{\gamma}_{m, k}^{*}\right)-\frac{\bar{\gamma}_{m, k}^{*}}{1+\bar{\gamma}_{m, k}^{*}} \log _{2} \bar{\gamma}_{m, k}^{*}$.

Then, the Dinkelbach method [26] is proposed to further transform the fractional objective function in (17) to a tractable non-fractional form. The novel optimization problem is written as follows:

$$
\begin{aligned}
\max _{\mathbf{P} \succ 0} & =\bar{R}^{*}(\mathbf{P})-t \cdot\left(P_{c}+\sum_{m=1}^{M} \sum_{k=1}^{K} p_{m, k}\right) \\
\text { s.t. } \quad & C 1: \sum_{m=1}^{M} \sum_{k=1}^{K} p_{m, k}\left(\bar{f}_{m, j}+\varepsilon_{j}\right) \leq I_{t h r} \\
& C 2: \bar{r}_{m, k}^{*} \geq R_{\min } \\
& C 3: \sum_{k=1}^{K} p_{m, k} \leq P_{\max } \\
& C 4: p_{m, k} \geq 0
\end{aligned}
$$


where the parameter $t$ is introduced to measure the weight of sum rate of SCUs and total power assumption. The definition of the novel function is given as

$$
F(t)=\max _{\mathbf{P} \succ 0}\left\{\bar{R}^{*}(\mathbf{P})-t \cdot\left(P_{c}+\sum_{m=1}^{M} \sum_{k=1}^{K} p_{m, k}\right)\right\}
$$

The solution of function (19) is equivalent to find the maximum EE to make the nonlinear equation $F(t)=0$. The detailed iterative sub-optimal power allocation scheme is illustrated in

Algorithm 1.

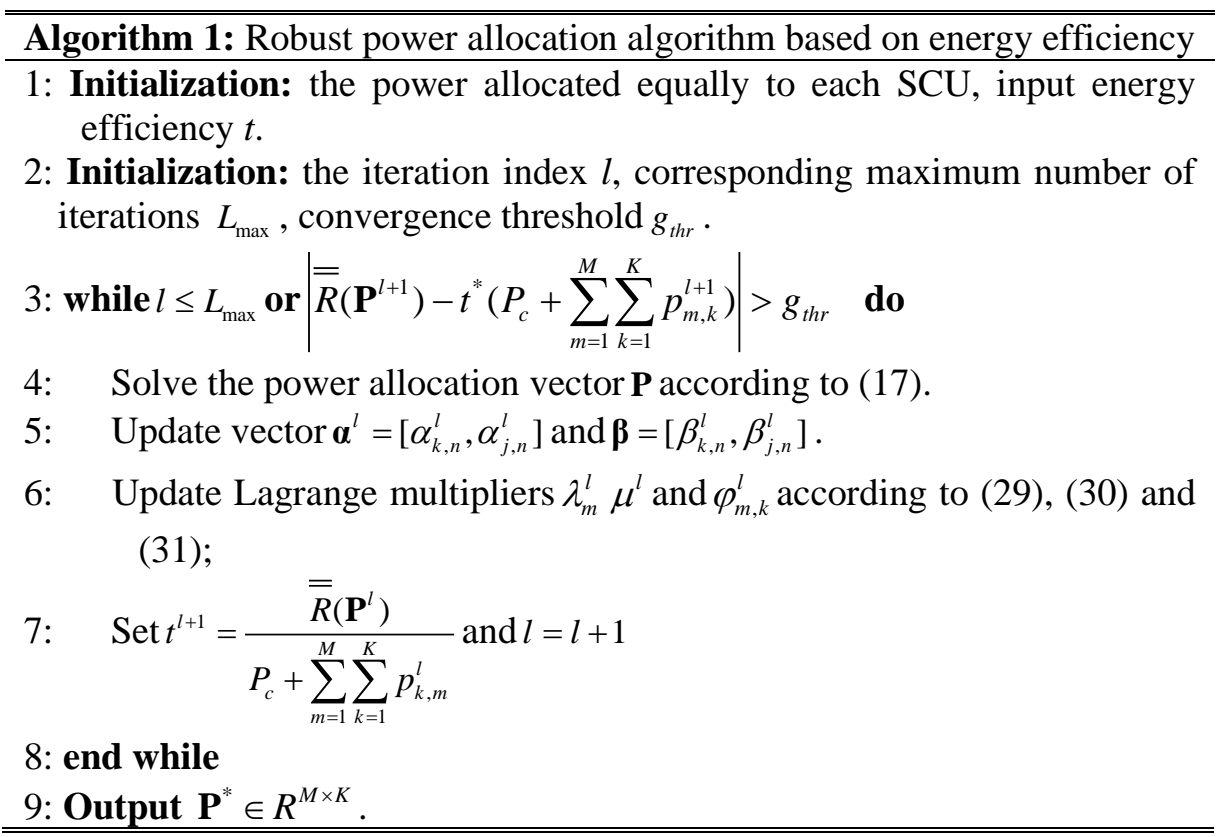

\subsection{The Close-Form of Power for SCUs}

After the transformation of optimization problem, it can be further solved by solving its Lagrange dual problem. The Lagrangian function corresponding to optimization problem (19) is expressed as

$$
\begin{aligned}
& L=\sum_{m=1}^{M} \sum_{k=1}^{K}\left(\alpha_{m, k} \log _{2} \bar{\gamma}_{m, k}+\beta_{m, k}\right)-t\left(P_{c}+\sum_{m=1}^{M} \sum_{k=1}^{K} p_{m, k}\right)+\lambda_{m}\left(P_{\max }-\sum_{k=1}^{K} p_{m, k}\right) \\
& +\mu\left[I_{t h r}-\sum_{m=1}^{M} \sum_{k=1}^{K} \sum_{j=1}^{J} p_{m, k}\left(\bar{f}_{m, j}+\varepsilon_{j}\right)\right]+\sum_{m=1}^{M} \sum_{k=1}^{K} \varphi_{m, k}\left(\alpha_{m, k} \log _{2} \bar{\gamma}_{m, k}+\beta_{m, k}-R_{\min }\right)
\end{aligned}
$$

where $\lambda_{m}, \mu$ and $\varphi_{m, k}$ are non-negative Lagrange multipliers. The defined dual function will be shown as follows:

$$
g(\lambda, \mu, \varphi)=\left\{\begin{array}{l}
\max _{\mathbf{P}} L(\mathbf{P}, \lambda, \mu, \varphi) \\
\text { s.t. } C 1, C 2, C 3, C 4
\end{array}\right.
$$

Furthermore, the dual problem of (20) can be written as

$$
\begin{aligned}
& \min _{\lambda, \mu, \boldsymbol{\varphi}} g(\boldsymbol{\lambda}, \mu, \boldsymbol{\varphi}) \\
& \text { s.t. } \boldsymbol{\lambda}>\mathbf{0}, \mu>0, \boldsymbol{\varphi}>\mathbf{0}
\end{aligned}
$$

Considering the complexity of calculation, it is assumed that there are two SCUs in the same small cell. The proposed algorithm is still applicable to the scenario where multiple SCUs are 
deployed in a small cell. The problem can be solved by finding the optimal $\mathbf{P}, \boldsymbol{\lambda}, \mu, \boldsymbol{\varphi}$. Based on the KKT conditions, the close-form of powers for SCUs can be derived as

$$
p_{m, 1}=\frac{-\left(-B_{m, 2}+C_{m, 1}-A_{m, 2} D_{m, 1}\right)-\sqrt{\left(-B_{m, 2}+C_{m, 1}+A_{m, 2} D_{m, 1}\right)^{2}+4 A_{m, 2} C_{m, 1} D_{m, 1}}}{-2 D_{m, 1}}
$$

where

$$
\begin{gathered}
B_{m, 2}=\frac{\left(1+\varphi_{m, 2}\right) \alpha_{m, 2}}{\ln 2} \\
C_{m, 1}=\frac{\left(1+\varphi_{m, 1}\right) \alpha_{m, 1}}{\ln 2} \\
D_{m, 1}=\mu\left(\bar{f}_{m, j}+\varepsilon_{j}\right)+t+\lambda_{m, 1}
\end{gathered}
$$

And the power of the other SCU in the $m$ th small cell can be derived as

$$
p_{m, 2}=\frac{\left(1+\varphi_{m, 2}\right) \alpha_{m, 2}}{\left[t+\lambda_{m, 2}+\mu\left(\bar{f}_{m, j}+\varepsilon_{j}\right)\right] \ln 2}
$$

Based on (24) and (28), the allocated power can be known in advance, and Since the fact that objective function of dual problem is differentiable, the Lagrange multipliers $\lambda_{m}, \mu$ and $\varphi_{m, k}$ can be obtained by the gradient descent method, respectively

$$
\begin{gathered}
\lambda_{m}{ }^{l+1}=\left[\lambda_{m}{ }^{l}-\delta_{1}^{l} \cdot\left(P_{\max }-\sum_{k=1}^{K} p_{m, k}\right)\right]^{+} \\
\mu^{l+1}=\left[\mu^{l}-\delta_{2}^{l} \cdot\left(I_{t h r}-\sum_{m=1}^{M} \sum_{k=1}^{K} \sum_{j=1}^{J} p_{m, k}\left(\bar{f}_{m, j}+\varepsilon_{j}\right)\right)\right]^{+} \\
\varphi_{m, k}^{l+1}=\left[\varphi_{m, k}^{l}-\delta_{3}^{l} \cdot\left(\alpha_{m, k} \log _{2} \gamma_{m, k}+\beta_{m, k}-R_{\min }\right)\right]^{+}
\end{gathered}
$$

where $l$ denotes the iteration index, and $\delta_{1}^{l}, \delta_{2}^{l}, \delta_{3}^{l}$ are the positive step sizes, respectively. It is known that the gradient descent method can ensure the iteration converges to the optimal Lagrange multipliers in terms of some sufficiently small step-size.

\subsection{The Convergence Performance Proof of Algorithm 1}

In here, we will prove the proposed algorithm 1 is convergent according to [7]. First, we can know that $F(t)$ in (20) is a monotonically decreasing function about $t$. The detailed certification process can be illustrated as follows:

Proof: we can assume that $P^{(1)}$ and $P^{(2)}$ are the optimally allocated power for $F\left(t^{(1)}\right)$ and $F\left(t^{(2)}\right)$, separately. We can obtain that

$$
\begin{aligned}
F\left(t^{(2)}\right) & =\max _{\mathbf{P} \succ 0}\left\{\bar{R}^{*}(\mathbf{P})-t^{(2)} \cdot\left(P_{c}+\sum_{m=1}^{M} \sum_{k=1}^{K} p_{m, k}\right)\right\} \\
& =\bar{R}^{*}\left(\mathbf{P}^{(2)}\right)-t^{(2)} \cdot\left(P_{c}+\sum_{m=1}^{M} \sum_{k=1}^{K} p_{m, k}^{(2)}\right) \\
& >\bar{R}^{*}\left(\mathbf{P}^{(1)}\right)-t^{(2)} \cdot\left(P_{c}+\sum_{m=1}^{M} \sum_{k=1}^{K} p_{m, k}^{(1)}\right) \\
& >\bar{R}^{*}\left(\mathbf{P}^{(1)}\right)-t^{(1)} \cdot\left(P_{c}+\sum_{m=1}^{M} \sum_{k=1}^{K} p_{m, k}^{(1)}\right)=F\left(t^{(1)}\right)
\end{aligned}
$$


Because $F\left(t^{(1)}\right)=\max _{\mathbf{P} \succ 0}\left\{\bar{R}^{*}(\mathbf{P})-t^{(1)} \cdot\left(P_{c}+\sum_{m=1}^{M} \sum_{k=1}^{K} p_{m, k}\right)\right\} \geq \bar{R}^{*}\left(\mathbf{P}^{(1)}\right)-t^{(1)} \cdot\left(P_{c}+\sum_{m=1}^{M} \sum_{k=1}^{K} p_{m, k}^{(1)}\right)=0$, it can be obtained that $F\left(t^{(1)}\right) \geq 0$. Then, we will give the convergence performance proof of proposed algorithm. The EE $t$ increases with the growth of the number of iterations. It is assumed that the optimal EE is $t^{*}$ and the corresponding power is $P^{*}$. We denote the achieved power allocation scheme in the $l$ th iteration is $P(l)$, and the corresponding $\mathrm{EE}$ in the $l$ th iteration and the $(l+1)$ th iteration is not optimal. According to (20), we can find that $F\left(t^{(l)}\right)>0$ and $F\left(t^{(l+1)}\right)>0$. And because $t^{(l+1)}=\frac{\bar{R}^{*}\left(\mathbf{P}^{(l)}\right)}{P_{c}+\sum_{m=1}^{M} \sum_{k=1}^{K} p_{m, k}^{(l)}}$, we can obtain that

$$
\begin{aligned}
F\left(t^{(l)}\right) & =\bar{R}^{*}\left(\mathbf{P}^{(l)}\right)-t^{(l)} \cdot\left(P_{c}+\sum_{m=1}^{M} \sum_{k=1}^{K} p_{m, k}^{(l)}\right) \\
& >t^{(l+1)} \cdot\left(P_{c}+\sum_{m=1}^{M} \sum_{k=1}^{K} p_{m, k}^{(l)}\right)-t^{(l)} \cdot\left(P_{c}+\sum_{m=1}^{M} \sum_{k=1}^{K} p_{m, k}^{(l)}\right) \\
& =\left(t^{(l+1)}-t^{(l)}\right) \cdot\left(P_{c}+\sum_{m=1}^{M} \sum_{k=1}^{K} p_{m, k}^{(l)}\right)>0
\end{aligned}
$$

Because $P_{c}+\sum_{m=1}^{M} \sum_{k=1}^{K} p_{m, k}^{(l)}>0$ is always satisfied, $F\left(t^{(l)}\right)$ will be equal to zero and we will find the optimal EE when the number of iteration is large enough.

\section{Simulation Results}

The output performance of SCUs can be shown in this section. In the simulations, SCUs and MCUs are deployed at random within the SBSs and MBSs, respectively. The radius of macro cell and small cell are $250 \mathrm{~m}$ and $30 \mathrm{~m}$, respectively and the transmission power of MBS and maximum transmission power constraints $P_{\max }$ of SBSs are $40 \mathrm{dBm}$ and $30 \mathrm{dBm}$, severally. The total system bandwidth is $1 \mathrm{MHz}$ and the noise power density is $-174 \mathrm{dBm} / \mathrm{Hz}$. Path-loss exponent is 3.76 and user minimum data rate is assumed as $0.6 \mathrm{bps} / \mathrm{Hz}$. Meanwhile, the upper boundary of uncertainty, $\varepsilon_{j}$, is equal to 0.05 .

\subsection{The Relationship between EE Performance of SCUs and $P_{\max }$}

Fig. 2 displays the EE of SCUs versus the maximum transmission power of SBS $P_{\max }$ with different power schemes which include the proposed robust power allocation algorithm (PRPAA) in NOMA and OFDMA system, and the equal power allocation scheme in NOMA system, where $\eta=0.05$ and $\delta=0.05$. From Fig. 2, we note that the EE performance of SCUs of PRPAA in NOMA and OFDMA systems increases with the increasing of $P_{\max }$, while the output performance of PRPAA in NOMA is obviously greater than that in OFDMA. Meanwhile, the EE of equal power allocation in NOMA scheme is worst in the three schemes. The reason is that when the power is greater than 23dbm, the gain of the rate is less than the increase of the power consumption, and the overall EE become smaller, which leads to the performance decrease. From that, we can prove the validity of the proposed algorithm. 


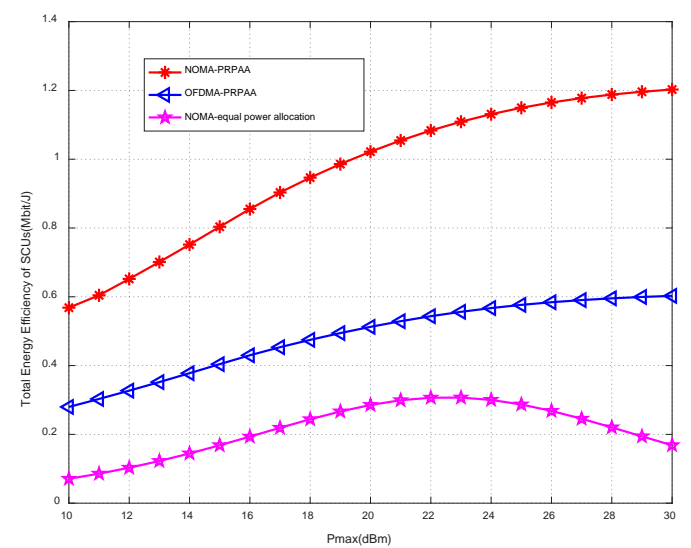

Fig. 2. the EE of SCUs versus the $P_{\max }$ with different algorithms

\subsection{The Convergence Performance of Proposed Algorithm}

The convergence rate of PRPAA is investigated versus different number of small cells shown in Fig. 3. In the convergence rate, the upper boundary values of uncertainty parameters $\eta$ and $\delta$ are set to be 0.05 and 0.05 , respectively. It can be seen that the EE with the small cells is stable after six times iteration, which demonstrates the proposed algorithm has a fast convergence rate. Furthermore, from Fig. 3, we can know that a higher EE can be achieved when more small cells exist. For example, as the number of small cells $M$ is equal to 11, the EE performance is $7.31 \%$ higher than that with $M=8$, and is $18.01 \%$ superior to that with $M=5$. The results also indicate that the convergence rate of the proposed algorithm has little correlation with the number of small cells. However, the number of small cells is 5, 8 or 11, the proposed algorithm can achieve good convergence performance after the fifth iteration.

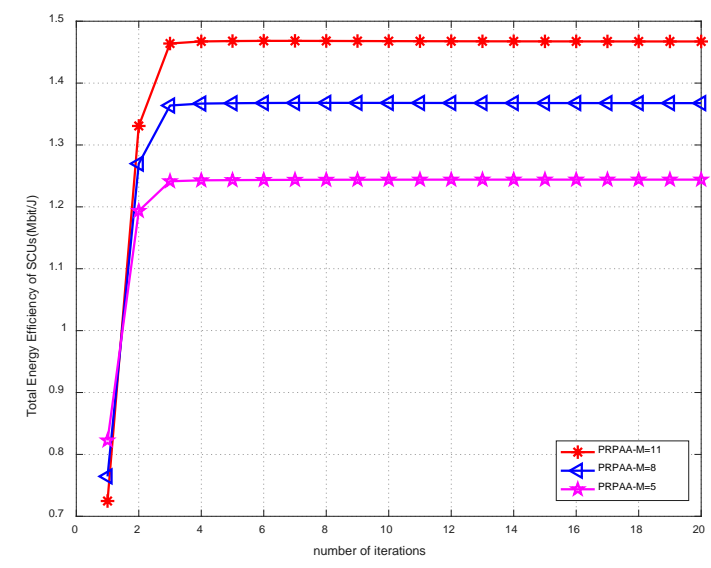

Fig. 3. the convergence performance of the proposed algorithm

\subsection{The Relationship between EE performance and Upper Bound of Channel Uncertainties}

Fig. 4 displays the EE performance of different algorithms versus the upper boundary of the uncertainty parameter $\delta$. The different algorithms include the PRPAA and the power allocation with exact estimation of channel state information (PAECSI) in NOMA system and traditional OFDMA system, respectively. From Fig. 4, we note that the EE performance of 
PRPAA decreases with the increasing of the parameter $\delta$, but EE performance of the proposed algorithm is close to that of the PAECSI in the same system. This is mainly because $\delta$ is considered in our algorithm and is dealt with the worst-case performance optimization. In addition, the PRPAA-NOMA scheme can obtain a higher EE performance than PRPAA-OFDMA scheme when the value of the parameter $\delta$ is the same.

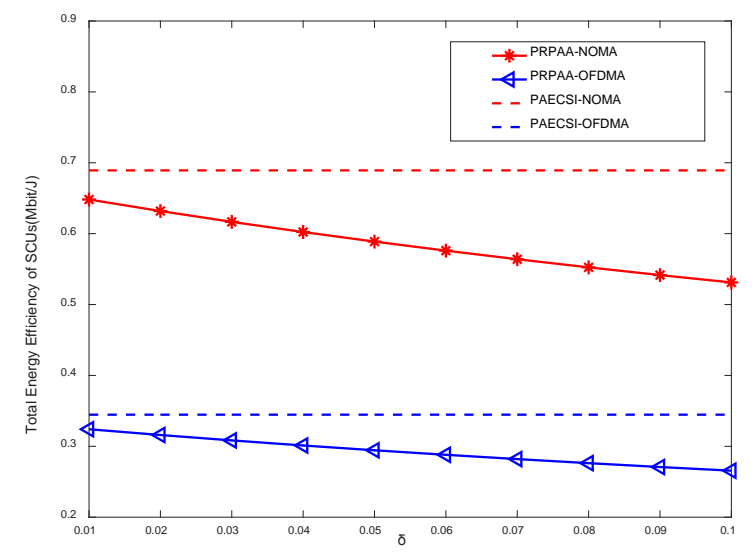

Fig. 4. the EE of SCUs versus the upper boundary of uncertainty $\delta$ with different algorithms

The output performance of PRPAA with different $\delta$ for NOMA HetNets versus the upper boundary of the uncertainty parameter $\eta$ is shown in Fig. 5 . The EE performance tends to decrease with the increasing of the parameter $\eta$. As expected, a better EE performance can be achieved when $\delta$ is small. In other words, the output performance downgrades with a bigger $\eta$ and $\delta$. For example, at $\eta=0.05$, the EE performance with $\delta=0.01$ is $10.87 \%$ larger than that with $\delta=0.05$, and is $23.61 \%$ larger than that with $\delta=0.1$. The reason is that the interference increases with the increasing of the parameters $\delta$ and $\eta$, which leads to the decrease of the EE performance. This indicates that the EE performance is better when the estimated value is closer to the actual value under imperfect CSI.

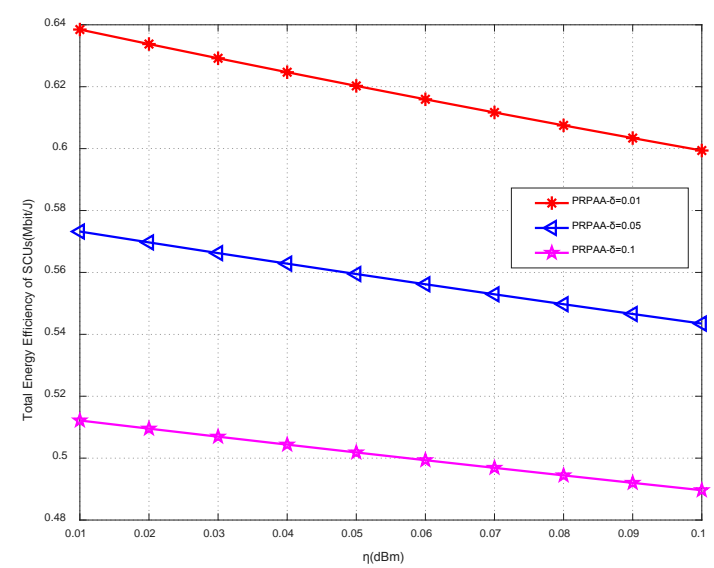

Fig. 5. the EE of SCUs versus the upper boundary of uncertainty with different $\delta$ 


\subsection{The Relationship between EE performance and the Number of Small Cells}

Fig. 6 compares the EE performance of different algorithms versus the number of small cells $M$ at $\eta=0.05$ and $\delta=0.05$. The difference algorithms involve the proposed PRPAA and the equal power allocation scheme in NOMA system and OFDMA system, respectively. In Fig. 6, it is shown that the equal power allocation algorithm may provide worse performance whether NOMA system or OFDMA system than PRPAA. From Fig. 6, We can also see that the EE performance of PRPAA increases with the growth of $M$, while the EE performance of equal power allocation algorithm decreases with the growth of $M$. The reason is that the inter-cell interference gets serious and the transmission power of each user-stream needs to be large enough to offset against the inter-cell interference, which leads to have a great negative impact on the transmission of NOMA HetNets. Furthermore, a better output performance can be achieved in NOMA system compared with OFDMA system with the same number of small cells.

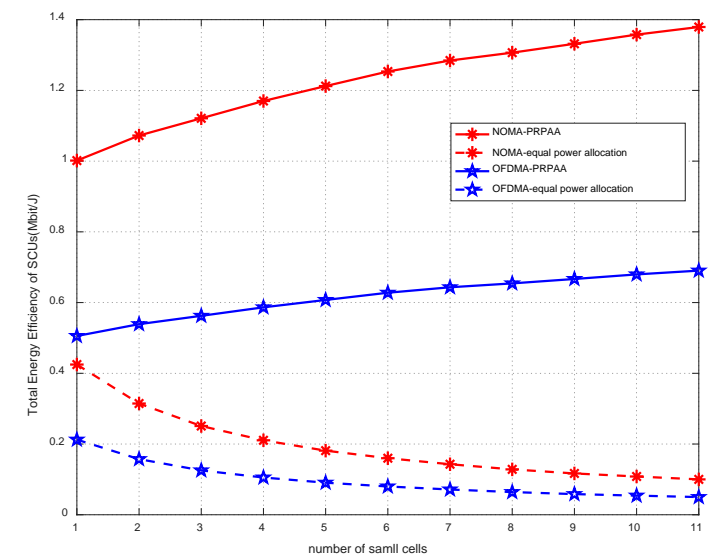

Fig. 6. the EE of SCUs versus the numbers of small cells with different algorithms

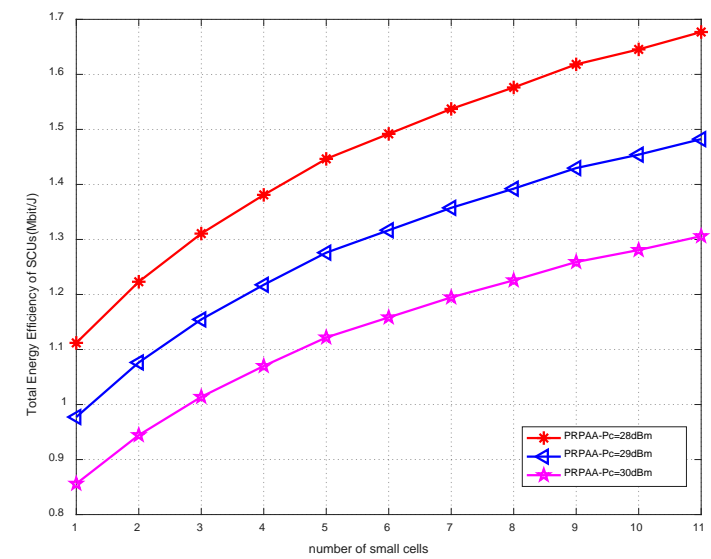

Fig. 7. impact of on total EE for different numbers of small cells

Fig. 7 shows the relationship between EE performance of the proposed PRPAA with the different power assumption $P_{c}$ and the number of small cells $M$. It can be seen that the proposed algorithm can improve the EE performance with the increasing of the number of small cells, and the increasing trend of EE performance becomes smaller as $M$ gets larger. This can be 
attributed to the fact that the in-tier interference is bigger when more small cells exist, so the EE performance cannot increase obviously when the number of users increases. Meanwhile the EE performance of the proposed algorithm degrades as the $P_{c}$ increases. For example, the EE with $P_{c}=28 \mathrm{dBm}$ is $13.29 \%$ superior to that with $P_{c}=29 \mathrm{dBm}$, and is $28.84 \%$ larger than that with $P_{c}=30 \mathrm{dBm}$ as the number of small cells is six.

\section{Conclusion}

In this paper, a power allocation scheme for a downlink NOMA HetNet is proposed with the imperfect CSI. The optimization problem is formulated based on EE under the consideration of QoS of MCUs and SCUs. Then, we transform the optimization problem to a robust optimization problem in terms of the channel gains uncertainty based on robust optimization theory and worst-case approach. The non-convex power allocation problem is solved through Dinkelbach method, sequential convex programming and the close-form of powers for SCUs are obtained via Lagrange dual approach. The output EE performance of the proposed scheme is better than that of traditional schemes and have a fast convergence performance.

\section{Reference}

[1] J. Chen, Y. Zhou and Y. Kuo, "Energy-efficiency resource allocation for cognitive heterogeneous networks with imperfect channel state information," IET Communications, vol. 10, no. 11, pp. 1312-1319, 2016. Article (CrossRef Link)

[2] F. Fang, H. Zhang, J. Cheng and V. C. M. Leung, "Energy-Efficient Resource Allocation for Downlink Non-Orthogonal Multiple Access Network," IEEE Trans. Wireless Commun., vol. 64, no. 9, pp. 3722-3732, Sep. 2016. Article (CrossRef Link)

[3] F. Wang, W. Chen, H. Tang and Q. Wu, "Joint Optimization of User Association, Subchannel Allocation, and Power Allocation in Multi-Cell Multi-Association OFDMA Heterogeneous Networks," IEEE Transactions on Communications, vol. 65, no. 6, pp. 2672-2684, June 2017. Article (CrossRef Link)

[4] K. Senel and M. Akar, "A Power Allocation Algorithm For Multi-Tier Cellular Networks With Heterogeneous QoS and Imperfect Channel Considerations," IEEE Trans. Wireless Commun, vol. 16, no. 11, pp. 7184-7194, Nov. 2017. Article (CrossRef Link)

[5] U. Siddique, H. Tabassum, E. Hossain and D. I. Kim, "Channel-Access-Aware User Association With Interference Coordination in Two-Tier Downlink Cellular Networks," IEEE Trans. Veh. Technol., vol. 65, no. 7, pp. 5579-5594, July 2016. Article (CrossRef Link)

[6] B. Di, L. Song and Y. Li, "Sub-Channel Assignment, Power Allocation, and User Scheduling for Non-Orthogonal Multiple Access Networks," IEEE Trans. Wireless Commun., vol. 15, no. 11, pp. 7686-7698, Nov. 2016. Article (CrossRef Link)

[7] F. Fang, H. Zhang, J. Cheng, S. Roy and V. C. M. Leung, "Joint User Scheduling and Power Allocation Optimization for Energy-Efficient NOMA Systems With Imperfect CSI," IEEE J. Sel. Areas Commun., vol. 35, no. 12, pp. 2874-2885, Dec. 2017. Article (CrossRef Link)

[8] J. Choi, "Power Allocation for Max-Sum Rate and Max-Min Rate Proportional Fairness in NOMA," IEEE Communications Letters, vol. 20, no. 10, pp. 2055-2058, Oct. 2016. Article (CrossRef Link)

[9] F. Wang, W. Chen, H. Tang and Q. Wu, "Joint Optimization of User Association, Subchannel Allocation, and Power Allocation in Multi-Cell Multi-Association OFDMA Heterogeneous Networks," IEEE Transactions on Communications, vol. 65, no. 6, pp. 2672-2684, June 2017. Article (CrossRef Link) 
[10] X. Wang, F. Zheng, P. Zhu and X. You, "Energy-Efficient Resource Allocation in Coordinated Downlink Multicell OFDMA Systems," IEEE Trans. Veh. Technol., vol. 65, no. 3, pp. 1395-1408, Mar. 2016. Article (CrossRef Link)

[11] F. Fang, J. Cheng and Z. Ding, "Joint Energy Efficient Subchannel and Power Optimization for a Downlink NOMA Heterogeneous Network," IEEE Trans. Veh. Technol., vol. 68, no. 2, pp. 1351-1364, Feb. 2019. Article (CrossRef Link)

[12] J. Zhao, Y. Liu, K. K. Chai, A. Nallanathan, Y. Chen and Z. Han, "Spectrum Allocation and Power Control for Non-Orthogonal Multiple Access in HetNets," IEEE Trans. Wireless Commun, vol. 16, no. 9, pp. 5825-5837, Sep. 2017. Article (CrossRef Link)

[13] Z. Song, Q. Ni and X. Sun, "Distributed Power Allocation for Nonorthogonal Multiple Access Heterogeneous Networks," IEEE Communications Letters, vol. 22, no. 3, pp. 622-625, March 2018. Article (CrossRef Link)

[14] B. Xu, Y. Chen, J. R. Carrión and T. Zhang, "Resource Allocation in Energy-Cooperation Enabled Two-Tier NOMA HetNets Toward Green 5G," IEEE J. Sel. Areas Commun., vol. 35, no. 12, pp. 2758-2770, Dec. 2017. Article (CrossRef Link)

[15] S. Mallick, R. Devarajan, M. M. Rashid and V. K. Bhargava, "Resource Allocation for Selective Relaying Based Cellular Wireless System with Imperfect CSI," IEEE Transactions on Communications, vol. 61, no. 5, pp. 1822-1834, May 2013. Article (CrossRef Link)

[16] J. Chen, Y. Zhou and Y. Kuo, "Energy-efficiency resource allocation for cognitive heterogeneous networks with imperfect channel state information," IET Communications, vol. 10, no. 11, pp. 1312-1319, 2016. Article (CrossRef Link)

[17] Z. Wei, D. W. K. Ng, J. Yuan and H. Wang, "Optimal Resource Allocation for Power-Efficient MC-NOMA With Imperfect Channel State Information," IEEE Trans. Wireless Commun., vol. 65, no. 9, pp. 3944-3961, Sep. 2017. Article (CrossRef Link)

[18] Y. Xu, Y. Hu, G. Li and H. Zhang, "Robust resource allocation for heterogeneous wireless network: a worst-case optimisation," IET Communications, vol. 12, no. 9, pp. 1064-1071, 2018. Article (CrossRef Link)

[19] M. Hasan and E. Hossain, "Distributed Resource Allocation for Relay-Aided Device-to-Device Communication Under Channel Uncertainties: A Stable Matching Approach," IEEE Transactions on Communications, vol. 63, no. 10, pp. 3882-3897, Oct. 2015. Article (CrossRef Link)

[20] K. Zhu, E. Hossain and A. Anpalagan, "Downlink Power Control in Two-Tier Cellular OFDMA Networks Under Uncertainties: A Robust Stackelberg Game," IEEE Transactions on Communications, vol. 63, no. 2, pp. 520-535, Feb. 2015. Article (CrossRef Link)

[21] Y. Xu and X. Zhao, "Robust adaptive power control for cognitive radio networks," IET Signal Processing, vol. 10, no. 1, pp. 19-27, 2016. Article (CrossRef Link)

[22] S. Sun, W. Ni and Y. Zhu, "Robust Power Control in Cognitive Radio Networks: A Distributed Way," in Proc. of the 2011 IEEE International Conference on Communications (ICC), Kyoto, pp. 1-6, 2011. Article (CrossRef Link)

[23] X. Song, L. Dong, J. Wang, L. Qin and X. Han, "Energy Efficient Power Allocation for Downlink NOMA Heterogeneous Networks with Imperfect CSI," IEEE Access, vol. 7, pp. 39329-39340, 2019. Article (CrossRef Link)

[24] A. J. G. Anandkumar, A. Anandkumar, S. Lambotharan and J. A. Chambers, "Robust Rate Maximization Game Under Bounded Channel Uncertainty," IEEE Transactions on Vehicular Technology, vol. 60, no. 9, pp. 4471-4486, Nov. 2011. Article (CrossRef Link)

[25] J. Papandriopoulos and J. S. Evans, "SCALE: A Low-Complexity Distributed Protocol for Spectrum Balancing in Multiuser DSL Networks," IEEE Transactions on Information Theory, vol. 55, no. 8, pp. 3711-3724, Aug. 2009. Article (CrossRef Link)

[26] Stephen Boyd L V, Stephen Boyd L V, “Convex optimization,” IEEE Transactions on Automatic Control, vol. 51, no. 11, pp. 1859-1859, 2006. Article (CrossRef Link) 


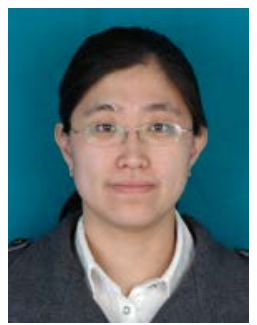

Xin Song was born in Jilin, China, in 1978. She received her B.S. degree from Northeastern University, China, in 2002, and M.S. degree from Northeastern University, China, in 2005. Her Ph.D degree is obtained in Communication and Information System in Northeastern University, China, in 2008. She is now working as a professor of Northeastern University at Qinhuangdao, China. Her research interests are in the area of robust adaptive beamforming and wireless communication.

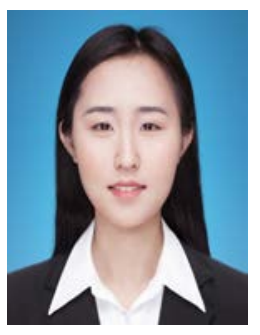

LI Dong was born in Liaoning Province, China, in 1995. She received the B.S. degree in Communication Engineering from Shenyang University of Technology, Shenyang, in 2017. She is currently pursuing M.S degree with Engineering Optimization and Smart Antenna Institute from Northeastern University, China. Her research interests include heterogeneous networks, non-orthogonal multiple access technology, and wireless resource allocation.

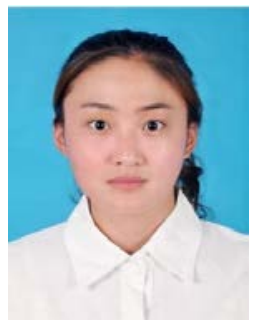

XUE Huang was born in Shandong Province, China, in 1994. She received the B.S. degree in Communication Engineering from Nanyang institute of technology, Nanyang, in 2017. She is currently pursuing M.S degree with Engineering Optimization and Smart Antenna Institute from Northeastern University. Her research interests include non-orthogonal multiple access, imperfect channel state information and resource allocation in heterogeneous wireless networks.

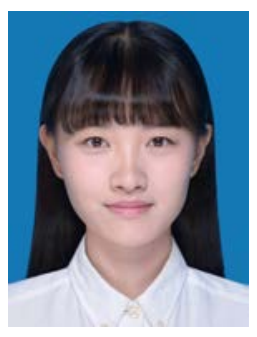

Lei Qin was born in Jilin Province, China, in 1994. She received the B.S. degree from the Qingdao University, Qingdao, in 2017. She is currently pursuing M.S degree with Engineering Optimization and Smart Antenna Institute from Northeastern University. Her research interests include user scheduling and resource allocation in heterogeneous wireless networks.

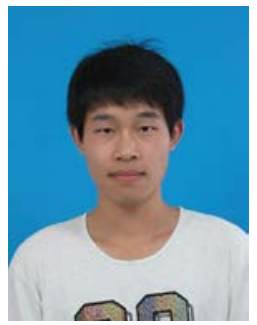

Xiuwei Han was born in Shandong Province, China, in 1995. He received the B.S. degree from the Binzhou University (BZU), Binzhou, in 2017. He is currently pursuing M.S degree with Engineering Optimization and Smart Antenna Institute from Northeastern University. His research interests include device-to-device communications and wireless resource allocation. 